\title{
Bounds of the Pinsker and Fannes Types on the Tsallis Relative Entropy
}

\author{
Alexey E. Rastegin \\ Department of Theoretical Physics, Irkutsk State University, Gagarin Bv. 20, Irkutsk 664003, Russia
}

\begin{abstract}
Pinsker's and Fannes' type bounds on the Tsallis relative entropy are derived. The monotonicity property of the quantum $f$-divergence is used for its estimating from below. For order $\alpha \in(0,1)$, a family of lower bounds of Pinsker type is obtained. For $\alpha>1$ and the commutative case, upper continuity bounds on the relative entropy in terms of the minimal probability in its second argument are derived. Both the lower and upper bounds presented are reformulated for the case of Rényi's entropies. The Fano inequality is extended to Tsallis' entropies for all $\alpha>0$. The deduced bounds on the Tsallis conditional entropy are used for obtaining inequalities of Fannes' type.
\end{abstract}

Keywords: Tsallis entropy, Rényi entropy, Pinsker inequality, Fano inequality, Fannes inequality, trace distance, convexity

\section{INTRODUCTION}

Physical systems with long-range interactions, long-time memories, or fractal structures can hardly be treated within the traditional background of statistical physics. The Tsallis entropies have been widely adopted in this direction [19]. As a rule, stationary states of such systems are described by one-parametric extensions of the Zipf-Mandelbrot power-law distribution. Generalized entropies have also found use as alternate measures of an informational content. For instance, the entropic uncertainty principle has been expressed in terms of both the Tsallis [27, 35] and Rényi entropies [38, 48]. Studies of generalized entropies allow to treat properties of the standard entropy in more general setting. The connection between strong subadditivity of the von Neumann entropy and the Wigner-Yanase-Dyson conjecture is a remarkable example (see [22, 24] and references therein).

The relative entropy, or Kullback-Leibler divergence 25], is frequently used as a measure of statistical distinguishability. Csiszar's f-divergence [10, 11] and Petz's quasi-entropies [29, 31] are famous generalizations of the Kullback-Leibler measure to the classical and quantum cases, respectively. In both the cases, properties of the relative entropy are the subject of active research. So, the development of a standard background to generalized entropies is an important issue. Lower bounds of the Pinsker type on the classical $f$-divergences were given by Csiszár [9]. In this paper, the function in which the variational distance is substituted is not explicit. Gilardoni presented explicit lower bounds on the classical $f$-divergences including the case of Tsallis' relative entropy [20].

In the present paper, we deduce some lower and upper bounds on the Tsallis relative entropy. The obtained bounds are expressed in terms of the trace distance between two probability distributions or density operators. The paper is organized as follows. In Section [1] the main definitions are given. One consequence of the monotonicity of the quantum $f$-divergence is considered in Section III. A family of lower bounds on the Tsallis relative entropy of order $\alpha \in(0,1)$ is derived in Section IV] In their essence, these inequalities are one-parametric extensions of the Pinsker inequality. The case of Rényi relative entropy is considered as well. In Section $\mathrm{V}$, upper bounds on the Tsallis relative $\alpha$-entropy of two probability distributions in terms of the minimal probability in its second argument are obtained. Fano type upper bounds on the conditional Tsallis entropy are derived for all $\alpha>0$ in Section VI. As is shown, these bounds lead to generalized inequalities of Fannes type.

\section{DEFINITIONS AND NOTATION}

In the classical regime, we will consider probability distributions over the index set $\Omega$ of finite cardinality $N$. The trace distance between probability distributions $P=\{p(x)\}$ and $Q=\{q(x)\}$ are then defined as

$$
D(P, Q):=\frac{1}{2} \sum_{x \in \Omega}|p(x)-q(x)| .
$$

Let $\mathcal{L}(\mathcal{H})$ be the space of linear operators on finite-dimensional Hilbert space $\mathcal{H}$. We also use the notation $\mathcal{L}_{+}(\mathcal{H})$ to denote the set of positive semidefinite operators. For any operator $\mathrm{X}$, we put $|\mathrm{X}| \in \mathcal{L}_{+}(\mathcal{H})$ as a unique positive square root of $X^{*} X \geq 0$. The trace norm $\|X\|_{1}:=\operatorname{tr}|X|$ and the trace distance

$$
\mathrm{D}(\mathrm{X}, \mathrm{Y}):=\frac{1}{2}\|\mathrm{X}-\mathrm{Y}\|_{1} \equiv \frac{1}{2} \operatorname{tr}|\mathrm{X}-\mathrm{Y}|
$$

are widely used in both the mathematical physics and quantum information theory. Using the Ky Fan norms, the partitioned versions of the above measures can be adopted properly [33, 34]. By $\operatorname{ker}(\mathbf{X})$ and $\operatorname{ran}(\mathrm{X})$ we denote the 
kernel and the range of operator $\mathrm{X} \in \mathcal{L}(\mathcal{H})$. Eigenvalues of the operator $\mathrm{X}$ form the multi-set $\operatorname{spec}(\mathrm{X})$. For $\mathrm{X}, \mathrm{Y} \in \mathcal{L}(\mathcal{H})$, we define the Hilbert-Schmidt inner product by

$$
\langle\mathrm{X}, \mathrm{Y}\rangle_{\mathrm{hs}}:=\operatorname{tr}\left(\mathrm{X}^{*} \mathrm{Y}\right)
$$

For positive $\alpha \neq 1$, the Tsallis $\alpha$-entropy of probability distribution $P=\{p(x)\}$ is defined by [44]

$$
H_{\alpha}(P):=\frac{1}{1-\alpha}\left(\sum_{x \in \Omega} p(x)^{\alpha}-1\right) \equiv-\sum_{x \in \Omega} p(x)^{\alpha} \ln _{\alpha} p(x)
$$

where $\ln _{\alpha} z \equiv\left(z^{1-\alpha}-1\right) /(1-\alpha)$ is the $\alpha$-logarithm. The maximal value $\ln _{\alpha} N$ is reached by the uniform distribution, when $p(x)=1 / N$ for all $x \in \Omega$. The Shannon entropy $H_{1}(P)=-\sum_{x} p(x) \ln p(x)$ is recovered in the limit $\alpha \rightarrow 1$. By $h_{\alpha}(u)$ we denote the binary Tsallis $\alpha$-entropy, i.e.

$$
h_{\alpha}(u):=H_{\alpha}(\{u, 1-u\})=-u^{\alpha} \ln _{\alpha} u-(1-u)^{\alpha} \ln _{\alpha}(1-u)
$$

where $u \in[0,1]$. This function is clearly concave and obeys $h_{\alpha}(u)=h_{\alpha}(1-u)$. Other important one-parametric generalization is the Rényi entropy (see [11, 30]):

$$
R_{\alpha}(P):=\frac{1}{1-\alpha} \ln \left(\sum_{x \in \Omega} p(x)^{\alpha}\right) .
$$

The obvious formula $(1-\alpha) R_{\alpha}(P)=\ln \left[1+(1-\alpha) H_{\alpha}(P)\right]$ relates the entropies (2.4) and (2.6). The quantum analogs of these entropies are respectively defined as

$$
\begin{aligned}
& \mathrm{H}_{\alpha}(\boldsymbol{\rho}):=\frac{1}{1-\alpha}\left(\operatorname{tr}\left(\boldsymbol{\rho}^{\alpha}\right)-1\right), \\
& \mathrm{R}_{\alpha}(\boldsymbol{\rho}):=\frac{1}{1-\alpha} \ln \left[\operatorname{tr}\left(\boldsymbol{\rho}^{\alpha}\right)\right] .
\end{aligned}
$$

where $\operatorname{tr}(\boldsymbol{\rho})=1$. Additivity properties of quantum entropies are an important issue. Subadditivity of the quantum Tsallis entropy (2.7) for $\alpha>1$ has been conjectured by Raggio [32] and later proved by Audenaert [1]. This result has been extended to some of so-called unified entropies [36]. The subadditivity property was believed to be true for the Wigner-Yanase entropy, until counterexamples were given [21, 43. Meantime, it is sufficient for the subadditivity that the bipartite state is pure. Other sufficient conditions for subadditivity of the Wigner-Yanase entropy are obtained in [8].

The standard relative entropy of $P=\{p(x)\}$ to $Q=\{q(x)\}$ is defined as [11]

$$
H_{1}(P \| Q)=-\sum_{x \in \Omega} p(x) \ln \frac{q(x)}{p(x)} .
$$

For density operators $\boldsymbol{\rho}$ and $\boldsymbol{\sigma}$, the quantum relative entropy is expressed as [30]

$$
\mathrm{H}_{1}(\boldsymbol{\rho} \| \boldsymbol{\sigma}):= \begin{cases}\operatorname{tr}(\boldsymbol{\rho} \ln \boldsymbol{\rho}-\boldsymbol{\rho} \ln \boldsymbol{\sigma}), & \text { if } \operatorname{supp}(\boldsymbol{\rho}) \leq \operatorname{supp}(\boldsymbol{\sigma}) \\ +\infty, & \text { otherwise }\end{cases}
$$

By $\operatorname{supp}(\mathrm{A})$, we mean the support projection of $\mathrm{A} \in \mathcal{L}_{+}(\mathcal{H})$. Instead of the entry $\operatorname{supp}(\boldsymbol{\rho}) \leq \operatorname{supp}(\boldsymbol{\sigma})$, the $\operatorname{condition}$ $\operatorname{ker}(\boldsymbol{\sigma}) \subset \operatorname{ker}(\boldsymbol{\rho})$ can be written as well [41]. In the classical regime, the Tsallis relative $\alpha$-entropy is introduced by [5]

$$
H_{\alpha}(P \| Q):=-\sum_{x \in \Omega} p(x) \ln _{\alpha} \frac{q(x)}{p(x)}=\frac{1}{1-\alpha}\left(1-\sum_{x \in \Omega} p(x)^{\alpha} q(x)^{1-\alpha}\right) .
$$

Basic properties of this measure are discussed in [5, 17]. The Rényi relative entropy is defined as [1]

$$
R_{\alpha}(P \| Q):=\frac{-1}{1-\alpha} \ln \left(\sum_{x \in \Omega} p(x)^{\alpha} q(x)^{1-\alpha}\right)
$$

Gilardoni derived Pinsker's type inequalities for both the Tsallis and Rényi relative entropies [20]. Note that the Tsallis relative entropy is written in [20] with the denominator $\alpha(1-\alpha)$ instead of $(1-\alpha)$. 
Let us to extend the definition (2.11) to any positive-valued functions $A$ and $B$ on the finite set $\Omega$. For given set $A=\{a(x)\}$, we put the index subset $\Omega_{A}=\{x: a(x) \neq 0\}$ and its complement $\widetilde{\Omega}_{A}$. For $\alpha>1$, the "Tsallis relative $\alpha$-entropy" of $A=\{a(x)\}$ to $B=\{b(x)\}$ is defined as

$$
H_{\alpha}(A \| B):= \begin{cases}\frac{1}{\alpha-1}\left(\sum_{x \in \Omega_{A}} a(x)^{\alpha} b(x)^{1-\alpha}-\sum_{x \in \Omega_{A}} a(x)\right), & \Omega_{A} \subset \Omega_{B}, \\ +\infty, & \text { otherwise } .\end{cases}
$$

Omitting the second entry, we obtain the definition for $0<\alpha<1$. For any positive scalar $\lambda$, we have

$$
H_{\alpha}(\lambda A \| \lambda B)=\lambda H_{\alpha}(A \| B)
$$

i.e. it is a homogeneous function of degree one. For $\alpha \in(0,1)$ and density operators $\boldsymbol{\rho}$ and $\boldsymbol{\sigma}$, we define the Tsallis relative entropy as

$$
\mathrm{H}_{\alpha}(\boldsymbol{\rho} \| \boldsymbol{\sigma}):=\frac{1}{1-\alpha}\left(1-\operatorname{tr}\left(\boldsymbol{\rho}^{\alpha} \boldsymbol{\sigma}^{1-\alpha}\right)\right) .
$$

For $\alpha>1$, the right-hand side of (2.15) is well-defined whenever $\operatorname{supp}(\boldsymbol{\rho}) \leq \operatorname{supp}(\boldsymbol{\sigma})$. In the singular case, when $\operatorname{supp}(\boldsymbol{\rho}) \nless \operatorname{supp}(\boldsymbol{\sigma})$, the right-hand side of (2.15) is dealt similar to the standard relative entropy (2.10). Namely, relative entropies are defined to be $+\infty$. Extending (2.12) to the quantum case, we define

$$
\mathrm{R}_{\alpha}(\boldsymbol{\rho} \| \boldsymbol{\sigma}):=\frac{-1}{1-\alpha} \ln \left[\operatorname{tr}\left(\boldsymbol{\rho}^{\alpha} \boldsymbol{\sigma}^{1-\alpha}\right)\right]
$$

For these entropies, we have the formula

$$
(\alpha-1) \mathrm{R}_{\alpha}(\boldsymbol{\rho} \| \boldsymbol{\sigma})=\ln \left[1+(\alpha-1) \mathrm{H}_{\alpha}(\boldsymbol{\rho} \| \boldsymbol{\sigma})\right],
$$

and its classical variety. For $\alpha>1$ and $\mathrm{A}, \mathrm{B} \in \mathcal{L}_{+}(\mathcal{H})$, we also introduce

$$
\mathrm{H}_{\alpha}(\mathrm{A} \| \mathrm{B}):= \begin{cases}\frac{1}{\alpha-1}\left(\operatorname{tr}\left(\mathrm{A}^{\alpha} \mathrm{B}^{1-\alpha}\right)-\operatorname{tr}(\mathrm{A})\right), & \operatorname{ran}(\mathrm{A}) \subset \operatorname{ran}(\mathrm{B}) \\ +\infty, & \text { otherwise }\end{cases}
$$

\section{A CONSEQUENCE OF MONOTONICITY OF THE $f$-DIVERGENCE}

In this section, we discuss one result which will be used to obtain quantum bounds of Pinsker's type. The Tsallis relative entropy (2.11) is closely related to the Csiszár $f$-divergence [10]. Let $z \mapsto f(z)$ be a convex function on $z \in[0,+\infty)$ with $f(1)=0$. The Csiszár $f$-divergence of $P=\{p(x)\}$ from $Q=\{q(x)\}$ is defined as [10, 11]

$$
S_{f}(P \| Q):=\sum_{x \in \Omega} q(x) f\left(\frac{p(x)}{q(x)}\right) .
$$

Taking $f_{\alpha}(z)=\left(z^{\alpha}-z\right) /(\alpha-1)$ with positive $\alpha \neq 1$, the formula (3.1) leads to (2.11). The standard case is recovered with $f(z)=z \ln z$. The definition (3.1) can generally be used without the normalization condition.

In the following, we use the convention that powers of a positive semidefinite operator are taken only on its support. So, by $A^{-1}$ and $A^{0}$ we respectively mean the generalized inverse and the support projection of $A$. $A$ quantum counterpart of Csiszár's $f$-divergence is introduced as follows [22]. For an operator $\mathrm{A} \in \mathcal{L}_{+}(\mathcal{H})$, let $\Lambda_{\mathrm{A}}$ and $\Upsilon_{\mathrm{A}}$ denote the left and the right multiplications by $\mathrm{A}$, respectively, defined as

$$
\Lambda_{\mathrm{A}}: \mathrm{X} \mapsto \mathrm{AX}, \quad \Upsilon_{\mathrm{A}}: \mathrm{X} \mapsto \mathrm{XA}, \quad \mathrm{X} \in \mathcal{L}(\mathcal{H})
$$

Left and right multiplications commute with each other, namely $\Lambda_{\mathrm{A}} \Upsilon_{\mathrm{B}}=\Upsilon_{\mathrm{B}} \Lambda_{\mathrm{A}}$ for $\mathrm{A}, \mathrm{B} \in \mathcal{L}+(\mathcal{H})$. Let $z \mapsto f(z)$ be a continuous function on $z \in[0,+\infty)$. Taking the set $\left\{a b^{-1}: a \in \operatorname{spec}(\mathrm{A}), b \in \operatorname{spec}(\mathrm{B})\right\}$, we write [22]

$$
f\left(\Lambda_{\mathrm{A}} \Upsilon_{\mathrm{B}^{-1}}\right):=\sum_{a \in \operatorname{spec}(\mathrm{A})} \sum_{b \in \operatorname{spec}(\mathrm{B})} f\left(a b^{-1}\right) \Lambda_{\mathrm{P}_{a}} \Upsilon_{\mathrm{Q}_{b}}
$$

where the formulas $\mathrm{A}=\sum_{a} a \mathrm{P}_{a}$ and $\mathrm{B}=\sum_{b} b \mathrm{Q}_{b}$ respectively express the spectral decompositions of $\mathrm{A}$ and $\mathrm{B}$. If $\operatorname{ran}(\mathrm{A}) \subset \operatorname{ran}(\mathrm{B})$, then the $f$-divergence of $A$ with respect to $B$ is defined as [22]

$$
\mathrm{S}_{f}(\mathrm{~A} \| \mathrm{B}):=\left\langle\mathrm{B}^{1 / 2}, f\left(\Lambda_{\mathrm{A}} \Upsilon_{\mathrm{B}^{-1}}\right) \mathrm{B}^{1 / 2}\right\rangle_{\mathrm{hs}}
$$


Let $\mathbb{1}$ be the identity operator. In general case, the quantum $f$-divergence is defined by the formula

$$
\mathrm{S}_{f}(\mathrm{~A} \| \mathrm{B}):=\lim _{\varepsilon \searrow 0} \mathrm{~S}_{f}(\mathrm{~A} \| \mathrm{B}+\varepsilon \mathbb{1}) .
$$

Basic properties of the quantity (3.4) are discussed in the paper [22]. Using the function $f_{\alpha}(z)=\left(z^{\alpha}-z\right) /(\alpha-1)$, we actually obtain the quantity (2.18). One of the most important properties of relative entropies is their monotonicity under the action of trace-preserving completely positive (TPCP) maps [45]. For general discussion of a role of stochastic maps in quantum theory, see the paper [7]. Many fundamental results of quantum information theory are closely related to the monotonicity of the standard relative entropy [24, 28, 46]. General conditions for the monotonicity of the quantum $f$-divergence are obtained in [22]. If the map $\Phi$ is TPCP-map and the function $f$ is operator convex on $[0,+\infty)$ then

$$
\mathrm{S}_{f}(\Phi(\mathrm{A}) \| \Phi(\mathrm{B})) \leq \mathrm{S}_{f}(\mathrm{~A} \| \mathrm{B}) .
$$

Note that the inequality (3.6) has generally been established in [22] under weaker conditions on the maps. From the monotonicity (3.6) we can derive simple upper bounds on the quantum $f$-divergence in terms of classical one. Let $\Pi \in \mathcal{L}_{+}(\mathcal{H})$ be a projection. It is known that the linear map

$$
\mathrm{X} \mapsto\{\operatorname{tr}(\Pi \mathrm{X}), \operatorname{tr}[(\mathbb{1}-\Pi) \mathrm{X}]\}
$$

is both trace-preserving and completely positive. Combining this fact with the monotonicity (3.6), we have arrived at a conclusion.

Lemma 1 Suppose $\mathrm{A}, \mathrm{B} \in \mathcal{L}_{+}(\mathcal{H})$. Let $\Pi_{ \pm} \in \mathcal{L}_{+}(\mathcal{H})$ obey $\Pi_{+}+\Pi_{-}=\mathbb{1}$ and be projectors onto the eigenspaces corresponding to positive and negative eigenvalues of $(\mathrm{A}-\mathrm{B})$, respectively. If the function $f$ is operator convex then

$$
\mathrm{S}_{f}(\mathrm{~A} \| \mathrm{B}) \geq S_{f}\left(\left\{u_{ \pm}^{\prime}\right\} \|\left\{v_{ \pm}^{\prime}\right\}\right)
$$

where $u_{ \pm}^{\prime}=\operatorname{tr}\left(\Pi_{ \pm} \mathrm{A}\right)$ and $v_{ \pm}^{\prime}=\operatorname{tr}\left(\Pi_{ \pm} \mathrm{B}\right)$.

The function $z \mapsto z^{\alpha}$ is operator concave on $\mathcal{L}_{+}(\mathcal{H})$ for $0 \leq \alpha \leq 1$ and operator convex on $\mathcal{L}_{+}(\mathcal{H})$ for $1 \leq \alpha \leq 2$ (see, respectively, theorems 4.2.3 and 1.5.8 in [4]). So the function $f_{\alpha}(z)=\left(z^{\alpha}-z\right) /(\alpha-1)$ is operator convex for $\alpha \in[0,2]$ and $\alpha \neq 1$. Combining this with the inequality (3.8) gives

$$
\mathrm{H}_{\alpha}(\mathrm{A} \| \mathrm{B}) \geq H_{\alpha}\left(\left\{u_{ \pm}^{\prime}\right\} \|\left\{v_{ \pm}^{\prime}\right\}\right)
$$

Up to a notation, the result (3.9) with density operators was presented in [39] (see theorem IV.1 therein). In the next section, we will use the relations (3.9) and

$$
\|\mathrm{A}-\mathrm{B}\|_{1}=\left|u_{+}^{\prime}-v_{+}^{\prime}\right|+\left|u_{-}^{\prime}-v_{-}^{\prime}\right|
$$

for estimating the right-hand of (3.8) from below in terms of the distance $\|\mathrm{A}-\mathrm{B}\|_{1}$.

\section{PINSKER TYPE INEQUALITIES FOR $\alpha \in(0,1)$}

Studies of distinguishability measures and relations between them is an actual issue of quantum information theory. The Pinsker inequality [10] and its quantum analog expressed as [23]

$$
\mathrm{H}_{1}(\boldsymbol{\rho} \| \boldsymbol{\sigma}) \geq 2 \mathrm{D}(\boldsymbol{\rho}, \boldsymbol{\sigma})^{2},
$$

are well-known results of such a kind. Lower and upper bounds on the relative entropy (2.10) were given in [2]. Upper bounds of the papers [2, 3] are similar to Fannes' inequality [12]. The authors of [42] proved that

$$
\mathrm{H}_{\alpha}(\boldsymbol{\rho} \| \boldsymbol{\sigma}) \leq \mathrm{H}_{1}(\boldsymbol{\rho} \| \boldsymbol{\sigma}) \leq \mathrm{H}_{\beta}(\boldsymbol{\rho} \| \boldsymbol{\sigma}),
$$

where $0 \leq \alpha<1$ and $1<\beta \leq 2$. So, for $1<\beta \leq 2$ the relative entropy $\mathrm{H}_{\beta}(\boldsymbol{\rho} \| \boldsymbol{\sigma})$ is bounded from below by the right-hand side of Eq. (4.1). More detailed lower bounds on the relative entropy (2.10) are presented in [2]. By (4.2), these lower bounds are all valid for $\mathrm{H}_{\beta}(\boldsymbol{\rho} \| \boldsymbol{\sigma})$ with $1<\beta \leq 2$. 
Let $\Pi_{+}$be a projector on the eigenspace corresponding to positive eigenvalues of the difference $(\boldsymbol{\rho}-\boldsymbol{\sigma})$. For normalized density operators, the inequality (3.9) together with the definitions (2.11) and (2.15) leads to the bound

$$
\mathrm{H}_{\alpha}(\boldsymbol{\rho} \| \boldsymbol{\sigma}) \geq H_{\alpha}(\{u, 1-u\}||\{v, 1-v\}),
$$

where we write $u=\operatorname{tr}\left(\Pi_{+} \boldsymbol{\rho}\right)$ and $v=\operatorname{tr}\left(\Pi_{+} \boldsymbol{\sigma}\right)$ for brevity. Denoting $t=|u-v|$, we also have $\|\boldsymbol{\rho}-\boldsymbol{\sigma}\|_{1}=2 t$ and $\mathrm{D}(\boldsymbol{\rho}, \boldsymbol{\sigma})=t$. In the paper [39], for $u, v \in[0,1]$ we have proved the inequality (see lemma IV.2 therein)

$$
\sqrt{u v}+\sqrt{(1-u)(1-v)} \leq \sqrt{1-t^{2}}
$$

whence $H_{1 / 2}(\{u, 1-u\} \|\{v, 1-v\}) \geq 2\left(1-\sqrt{1-t^{2}}\right)$ and $H_{1 / 2}(\boldsymbol{\rho} \| \boldsymbol{\sigma}) \geq \mathrm{D}(\boldsymbol{\rho}, \boldsymbol{\sigma})^{2}$. Note that the result (4.4) is actually a special case of the inequality

$$
\mathrm{D}(\boldsymbol{\rho}, \boldsymbol{\sigma}) \leq \sqrt{1-F(\boldsymbol{\rho}, \boldsymbol{\sigma})^{2}}
$$

where $F(\boldsymbol{\rho}, \boldsymbol{\sigma})=\operatorname{tr}|\sqrt{\boldsymbol{\rho}} \sqrt{\boldsymbol{\sigma}}|$ is the fidelity of $\boldsymbol{\rho}$ and $\boldsymbol{\sigma}$. The formula (4.5) was proved by Fuchs and van der Graaf [15]. Using (4.4), we obtain the following statement.

Lemma 2 Let $u, v \in[0,1]$ and $g(t)=1-\sqrt{1-t^{2}}$. For $\alpha \in[0,1 / 2]$ and $t=|u-v|$, there holds

$$
u^{\alpha} v^{1-\alpha}+(1-u)^{\alpha}(1-v)^{1-\alpha} \leq 1-2 \alpha g(t) .
$$

Proof. For fixed $u$ and $v$, we define the function

$$
\Phi_{u v}(\alpha)=u^{\alpha} v^{1-\alpha}+(1-u)^{\alpha}(1-v)^{1-\alpha}+2 \alpha g(t)-1 .
$$

The claim (4.6) is equivalent to the inequality $\Phi_{u v}(\alpha) \leq 0$ for all $\alpha \in[0,1 / 2]$. First, we have $\Phi_{u v}(0) \leq 0$ obviously; second, $\Phi_{u v}(1 / 2) \leq 0$ because of (4.4). Third, $\Phi_{u v}(\alpha)$ is a convex function of the parameter $\alpha$. Indeed, for $u, v \neq 0,1$ we write down

$$
\frac{\partial^{2} \Phi_{u v}}{\partial \alpha^{2}}=u^{\alpha} v^{1-\alpha}\left(\ln \frac{u}{v}\right)^{2}+(1-u)^{\alpha}(1-v)^{1-\alpha}\left(\ln \frac{1-u}{1-v}\right)^{2} \geq 0
$$

If a convex function is negative at the end points of some interval, it is negative in this interval everywhere.

Combining the statement of Lemma 2 with (3.9) gives a lower bound of the Pinsker type on the Tsallis relative $\alpha$-entropy for $\alpha \in(0,1)$. We formulate it for two positive operators with equal traces.

Theorem 1 Let $\mathrm{A}, \mathrm{B} \in \mathcal{L}_{+}(\mathcal{H}), \operatorname{tr}(\mathrm{A})=\operatorname{tr}(\mathrm{B})=\theta, \mathrm{D}(\mathrm{A}, \mathrm{B})=\tau$ and $g(t)=1-\sqrt{1-t^{2}}$. For all $\alpha \in(0,1)$, there holds

$$
\mathrm{H}_{\alpha}(\mathrm{A} \| \mathrm{B}) \geq \varkappa_{\alpha} \theta g(\tau / \theta)
$$

where the factor $\varkappa_{\alpha}=2 \alpha(1-\alpha)^{-1}$ for $0<\alpha \leq 1 / 2$ and $\varkappa_{\alpha}=2$ for $1 / 2 \leq \alpha<1$.

Proof. Using the notation of Lemma 1, we have $u_{+}^{\prime}+u_{-}^{\prime}=v_{+}^{\prime}+v_{-}^{\prime}=\theta$ and $\|\mathrm{A}-\mathrm{B}\|_{1}=2\left|u_{+}^{\prime}-v_{+}^{\prime}\right|$, whence $\tau=\left|u_{+}^{\prime}-v_{+}^{\prime}\right|$. It follows from (3.9) and (2.13) that

$$
\begin{aligned}
(1-\alpha) \mathrm{H}_{\alpha}(\mathrm{A} \| \mathrm{B}) & \geq(1-\alpha) H_{\alpha}\left(\left\{u_{ \pm}^{\prime}\right\} \|\left\{v_{ \pm}^{\prime}\right\}\right) \\
& =\theta\left[1-u^{\alpha} v^{1-\alpha}-(1-u)^{\alpha}(1-v)^{1-\alpha}\right],
\end{aligned}
$$

where $u=u_{+}^{\prime} / \theta, v=v_{+}^{\prime} / \theta$, and $|u-v|=\tau / \theta$. Due to (4.6), for $0<\alpha \leq 1 / 2$ the right-hand side of (4.10) is not less than $2 \alpha \theta g(\tau / \theta)$. Hence the claim (4.9) with $\varkappa_{\alpha}=2 \alpha(1-\alpha)^{-1}$ follows. For $1 / 2 \leq \alpha<1$, we put $\beta=1-\alpha$ and further write

$$
\beta \mathrm{H}_{\alpha}(\boldsymbol{\rho} \| \boldsymbol{\sigma}) \geq \theta\left[1-u^{1-\beta} v^{\beta}-(1-u)^{1-\beta}(1-v)^{\beta}\right] \geq 2 \beta \theta g(\tau / \theta) .
$$

Hence the claim (4.9) with $\varkappa_{\alpha}=2$ follows

For probability distributions, the lower bound (4.9) is rewritten with the classical trace distance $\tau=D(P, Q)$. Expanding the function $g(\tau / \theta)$ into power series, we obtain a family of lower bounds of the Pinsker type. Namely, we have the bound

$$
\mathrm{H}_{\alpha}(\mathrm{A} \| \mathrm{B}) \geq \varkappa_{\alpha} \sum_{n=1}^{\infty}\left(\begin{array}{c}
1 / 2 \\
n
\end{array}\right)(-1)^{n+1} \frac{\tau^{2 n}}{\theta^{2 n-1}}
$$


including $\mathrm{H}_{\alpha}(\mathrm{A}|| \mathrm{B}) \geq(2 \theta)^{-1} \varkappa_{\alpha} \tau^{2}$. The coefficient $\left(\begin{array}{c}1 / 2 \\ n\end{array}\right)(-1)^{n+1}$ is positive for all $n$. So, any partial sum of the series (4.12) provides a lower bound. In general, this series does not provide an expansion with the best constants at powers of the trace distance. For the standard relative entropy $H_{1}(P \| Q)$, such constants have been the subject of long-time research (see [14] and references therein). Using $f(z)=\left(z^{\alpha}-z\right) /(\alpha(\alpha-1))$, Gilardoni [20] obtained Pinsker's bound on the $f$-divergence (3.1) for $\alpha \in[-1,2]$ and $\alpha \neq 0,1$. When $\alpha \in[0,2]$, the results of the paper [20] can directly be combined with (3.8), since the quantum relative $\alpha$-entropy is monotone here. In our notation for the Tsallis case, the Pinsker type bound of [20] reads

$$
\mathrm{H}_{\alpha}(\boldsymbol{\rho} \| \boldsymbol{\sigma}) \geq 2 \alpha \tau^{2}+\frac{2}{9} \alpha(\alpha+1)(2-\alpha) \tau^{4},
$$

where $\tau=\mathrm{D}(\boldsymbol{\rho}, \boldsymbol{\sigma})$ is the trace distance between normalized density matrices. It must be stressed that the inequality (4.9) is better in some joint region of the trace distance and $\alpha$ close to $1 / 2$. Say, for $\alpha=1 / 2$ the bound (4.9) is stronger for all $\tau \neq 0$. In this case, we obtain from (4.12) that

$$
\mathrm{H}_{1 / 2}(\boldsymbol{\rho} \| \boldsymbol{\sigma}) \geq \tau^{2}+\frac{1}{4} \tau^{4}+\sum_{n=3}^{\infty}\left(\begin{array}{c}
1 / 2 \\
n
\end{array}\right)(-1)^{n+1} \tau^{2 n},
$$

whereas the bound (4.13) involves only the first two terms of the right-hand side of (4.14).

For $\alpha \in(0,1)$, we can also combine (2.17) with (4.9) and hence obtain

$$
\mathrm{R}_{\alpha}(\boldsymbol{\rho} \| \boldsymbol{\sigma}) \geq \frac{1}{\alpha-1} \ln \left[1-(1-\alpha) \varkappa_{\alpha} g(\tau)\right] \geq \varkappa_{\alpha} g(\tau),
$$

where $\tau=\mathrm{D}(\boldsymbol{\rho}, \boldsymbol{\sigma})$. Indeed, the function $x \mapsto(\alpha-1)^{-1} \ln [1-(1-\alpha) x]$ increases with $x \in\left[0, \frac{1}{1-\alpha}\right)$ and $-\ln (1-\xi) \geq \xi$ for $\xi \in[0,1)$. The inequality (4.15) can be regarded as the bound of Pinsker's type on the Rényi relative entropy for $\alpha \in(0,1)$. Thus, we have obtained a family of lower bounds in terms of the trace distance on both the relative entropies (2.15) and (2.16).

\section{UPPER CONTINUITY BOUNDS FOR $\alpha>1$}

One of basic features of the standard relative entropy is its unboundedness. The relative $\alpha$-entropy enjoys the same for $\alpha>1$. So we may ask a behaviour of $H_{\alpha}(P \| Q)$ as the minimal probability in $Q$ goes to zero. Of course, in the quantum case this question is more difficult due to the non-commutativity. For the standard relative entropy, such an upper bound was obtained in [6], and more bounds were given in [2, [3]. For the quantum relative $\alpha$-entropy of order $\alpha>1$, upper bounds in terms of the minimal eigenvalue of its second entry were obtained in [37]. It turns out that in the commutative case these bounds can be sharpened significantly. Our derivation will mainly based on the joint convexity. Namely, for each positive $\alpha \neq 1$ the quantity (2.13) satisfies

$$
H_{\alpha}\left(\theta A^{\prime}+(1-\theta) A^{\prime \prime} \| \theta B^{\prime}+(1-\theta) B^{\prime \prime}\right) \leq \theta H_{\alpha}\left(A^{\prime} \| B^{\prime}\right)+(1-\theta) H_{\alpha}\left(A^{\prime \prime} \| B^{\prime \prime}\right),
$$

for all $0 \leq \theta \leq 1$. This relation follows from the so-called "generalized log-sum inequality" (see (16) in [5]). The properties (2.14) and (5.1) lead to the following statement.

Lemma 3 Let $A, B, C$ be three sets of positive numbers, and let $\mathrm{A}, \mathrm{B}$, and $\mathrm{C}$ be three positive operators. There holds

$$
\begin{array}{lr}
H_{\alpha}(A+C \| B+C) \leq H_{\alpha}(A \| B) & (0 \leq \alpha<\infty), \\
\mathrm{H}_{\alpha}(\mathrm{A}+\mathrm{C} \| \mathrm{B}+\mathrm{C}) \leq \mathrm{H}_{\alpha}(\mathrm{A} \| \mathrm{B}) & (0 \leq \alpha \leq 2) .
\end{array}
$$

Proof. Using (2.14) and (5.1), we merely write

$$
\begin{aligned}
H_{\alpha}(A+C \| B+C) & =2 H_{\alpha}((A+C) / 2 \|(B+C) / 2) \\
& \leq H_{\alpha}(A \| B)+H_{\alpha}(C \| C)=H_{\alpha}(A \| B),
\end{aligned}
$$

since $H_{\alpha}(C \| C)=0$. The quantum relative entropy (2.18) also enjoys both the homogeneity of degree one and the joint convexity, but the latter only for $0 \leq \alpha \leq 2$ (see, e.g., the review [24]). Rewriting the above arguments with the quantum relative $\alpha$-entropy instead of the classical one, we have arrived at the claim (5.3). 
For the standard relative entropy (2.10), the relation (5.3) was proved in [2]. The inequality (5.2) can be utilized to obtain an upper bound on $H_{\alpha}(P \| Q)$ in terms of the trace distance $D(P, Q)$ and the minimal probability

$$
q_{0}:=\min \left\{q_{j}: j \in \Omega_{P}\right\} .
$$

Here we apply that any sum in $H_{\alpha}(P \| Q)$ is effectively restricted to the index subset $\Omega_{P}$. Defining the set $\Delta=P-Q$ with elements $\delta_{j}=p_{j}-q_{j}$, we put another set $\bar{Q}$ with positive elements

$$
\bar{q}_{j}:=\max \left\{q_{0},-\delta_{j}\right\}
$$

Writing $Q=\bar{Q}+(Q-\bar{Q})$ and using the property (5.2) with $C=Q-\bar{Q}$, we obtain

$$
H_{\alpha}(P \| Q)=H_{\alpha}(\Delta+\bar{Q}+(Q-\bar{Q}) \| \bar{Q}+(Q-\bar{Q})) \leq H_{\alpha}(\Delta+\bar{Q} \| \bar{Q}) .
$$

Use of (5.2) is correct here by positivity of both $\Delta+\bar{Q}$ and $Q-\bar{Q}$. Indeed, we have $\delta_{j}+\max \left\{q_{0},-\delta_{j}\right\} \geq 0$ and $q_{j}-\max \left\{q_{0}, q_{j}-p_{j}\right\} \geq 0$ due to (5.5). The maximization of the right-hand side of (5.7) is under the conditions $\sum_{j} \delta_{j}=0$ and $\sum_{j}\left|\delta_{j}\right|=2 D(P, Q)$. We separately consider the two cases, $D(P, Q) \leq q_{0}$ and $q_{0}<D(P, Q) \leq 1-q_{0}$.

Theorem 2 Let $q_{0}$ be defined by (5.5), $\Omega_{P} \subset \Omega_{Q}$ and $\tau=D(P, Q)$. For $\alpha>1$, the Tsallis relative $\alpha$-entropy is bounded from above as

$$
\begin{array}{lr}
H_{\alpha}(P \| Q) \leq \frac{1}{\alpha-1}\left(\left(q_{0}+\tau\right)^{\alpha} q_{0}^{1-\alpha}+\left(q_{0}-\tau\right)^{\alpha} q_{0}^{1-\alpha}-2 q_{0}\right) & \left(\tau \leq q_{0}\right), \\
H_{\alpha}(P \| Q) \leq \frac{1}{\alpha-1}\left(\left(q_{0}+\tau\right)^{\alpha} q_{0}^{1-\alpha}-\left(q_{0}+\tau\right)\right) & \left(q_{0}<\tau \leq 1-q_{0}\right) .
\end{array}
$$

Proof. It is convenient to define three subsets of the set $\Omega_{P}$ of cardinality $n$ :

$$
\begin{aligned}
& \omega_{x}:=\left\{j: j \in \Omega_{P}, 0<\delta_{j}\right\}, \\
& \omega_{y}:=\left\{j: j \in \Omega_{P},-q_{0} \leq \delta_{j}<0\right\}, \\
& \omega_{z}:=\left\{j: j \in \Omega_{P}, \delta_{j}<-q_{0}\right\} .
\end{aligned}
$$

We also introduce corresponding $n$-dimensional positive vectors $X, Y$, and $Z$. These vectors respectively have elements defined as

$$
x_{i}:=\left\{\begin{array}{cc}
\delta_{i}, & i \in \omega_{x} \\
0, & i \notin \omega_{x}
\end{array}, \quad y_{j}:=\left\{\begin{array}{cc}
-\delta_{j}, & j \in \omega_{y} \\
0, & j \notin \omega_{y}
\end{array}, \quad z_{k}:=\left\{\begin{array}{cc}
-\delta_{k}, & k \in \omega_{z} \\
0, & k \notin \omega_{z}
\end{array} .\right.\right.\right.
$$

Hence we write $\Delta=X-Y-Z$. By (5.6), there holds

$$
\bar{q}_{j}=\left\{\begin{array}{l}
q_{0}, \quad j \in \omega_{x} \cup \omega_{y} \\
z_{j}, \quad j \in \omega_{z}
\end{array}\right.
$$

Let us begin with the case $\tau \leq q_{0}$. Because of $\left|\delta_{j}\right| \leq \tau$, the set $\omega_{z}$ is empty here. We first assume that the numbers $\delta_{j}$ are all non-zero, whence $\Omega_{P}=\omega_{x} \cup \omega_{y}$. The conditions $\sum_{j} \delta_{j}=0$ and $\sum_{j}\left|\delta_{j}\right|=2 \tau$ are rewritten as

$$
\sum_{i \in \omega_{x}} x_{i}=\sum_{j \in \omega_{y}} y_{j}=\tau
$$

In terms of $x_{i}$ and $y_{j}$, the right-hand side of (5.7) is represented as the function

$$
F\left(x_{i}, y_{j}\right)=\frac{1}{\alpha-1}\left(\sum_{i \in \omega_{x}}\left(q_{0}+x_{i}\right)^{\alpha} q_{0}^{1-\alpha}+\sum_{j \in \omega_{y}}\left(q_{0}-y_{j}\right)^{\alpha} q_{0}^{1-\alpha}-n q_{0}\right) .
$$

Possible values of the variables $x_{i}$ and $y_{j}$ correspond to interior points of the simplex defined by the conditions $0 \leq x_{i}$, $0 \leq y_{j}$ and (5.15). Recall that the global maximum of a convex function relative to a convex set is reached at one of the extreme points of that set [40]. Hence the maximal value of $F\left(x_{i}, y_{j}\right)$ on the simplex is equal to the right-hand side of (5.8). It is reached when one of the $x_{i}$ 's and one of the $y_{j}$ 's are equal to $\tau$ and other are all zero. Of course, values of $F\left(x_{i}, y_{j}\right)$ in the interior points of the simplex do not exceed this maximum. If some of the $\delta_{j}$ 's are zero then the question is actually reduced to the above reasons, but with diminished $n$. 
In the case $q_{0}<\tau$, we suppose again that the numbers $\delta_{j}$ are all non-zero. Instead of (5.15), we have

$$
\sum_{i \in \omega_{x}} x_{i}=\sum_{j \in \omega_{y}} y_{j}+\sum_{k \in \omega_{z}} z_{k}=\tau .
$$

We first modify the right-hand side of (5.7). From (5.14), we have $\bar{Q}=q_{0}\left(I_{x}+I_{y}\right)+Z$ and

$$
\Delta+\bar{Q}=q_{0}\left(I_{x}+I_{y}\right)+X-Y .
$$

Here $I_{x}$ denote the indicator of the set $\omega_{x}$ taking the value 1 for $j \in \omega_{x}$ and 0 for $j \notin \omega_{x}$. Note that if the set $\Omega_{Z}$ does not intersect with both the $\Omega_{A}$ and $\Omega_{B}$ then $H_{\alpha}(A \| B+Z)=H_{\alpha}(A|| B)$. Using this fact twice and the inequality (5.2) again with positive $C=q_{0} I_{y}-Y$, we rewrite the right-hand side of (5.7) as

$$
\begin{aligned}
& H_{\alpha}\left(q_{0}\left(I_{x}+I_{y}\right)+X-Y \| q_{0}\left(I_{x}+I_{y}\right)+Z\right)=H_{\alpha}\left(q_{0} I_{x}+X+C \| q_{0} I_{x}+Y+C\right) \\
& \leq H_{\alpha}\left(q_{0} I_{x}+X \| q_{0} I_{x}+Y\right)=H_{\alpha}\left(q_{0} I_{x}+X \| q_{0} I_{x}\right) .
\end{aligned}
$$

The latter can be rewritten as the function

$$
G\left(x_{i}\right)=\frac{1}{\alpha-1}\left(\sum_{i \in \omega_{x}}\left(q_{0}+x_{i}\right)^{\alpha} q_{0}^{1-\alpha}-\left(n_{x} q_{0}+\tau\right)\right),
$$

where $n_{x}$ is cardinality of the $\omega_{x}$. Possible values of the variables $x_{i}$ relate to interior points of the simplex defined by $0 \leq x_{i}$ and $\sum_{i} x_{i}=\tau$. So the maximum of $G\left(x_{i}\right)$ is equal to the right-hand side of (5.9) and reached, when one of the $x_{i}$ 's is $\tau$ and other are all zero. As above, we reduce the case, in which some of the $\delta_{j}$ 's are zero.

The upper bounds (5.8) and (5.9) have a behavior $q_{0}^{1-\alpha}$ with respect to the minimal probability $q_{0}$. For the quantum relative entropy $\mathrm{H}_{\alpha}(\boldsymbol{\rho} \| \boldsymbol{\sigma})$, upper bounds with a similar dependence on the minimal eigenvalue of $\boldsymbol{\sigma}$ were obtained in [37]. The bounds (5.8) and (5.9) are stronger, but their proof is quite restricted to the commutative case. The principal point is that positivity of diagonal elements of a matrix do not imply positivity of matrix itself (except for the case of diagonal matrices). Note that the inequalities (5.8) and (5.9) can be rewritten in terms of $\alpha$-logarithm as

$$
\begin{aligned}
& H_{\alpha}(P \| Q) \leq-\left(q_{0}+\tau\right) \ln _{\alpha}\left(\frac{q_{0}}{q_{0}+\tau}\right)-\left(q_{0}-\tau\right) \ln _{\alpha}\left(\frac{q_{0}}{q_{0}-\tau}\right), \\
& H_{\alpha}(P \| Q) \leq-\left(q_{0}+\tau\right) \ln _{\alpha}\left(\frac{q_{0}}{q_{0}+\tau}\right),
\end{aligned}
$$

respectively for $\tau \leq q_{0}$ and $q_{0}<\tau \leq 1-q_{0}$. Using (2.17) in classical setting, the bounds (5.21) and (5.22) remain valid with $R_{\alpha}(P \| Q)$ instead of $H_{\alpha}(P \| Q)$. In fact, the function $x \mapsto \ln [1+(\alpha-1) x]$ increases with $x>0$ and $\ln (1+\xi) \leq \xi$ for $\xi \geq 0$. The upper bounds (5.21) and (5.22) are $\alpha$-parametric extensions of the bounds obtained for the standard relative entropy in [2].

\section{NOTES ON THE FANO AND FANNES INEQUALITIES}

In this section, we will obtain upper bounds on the conditional Tsallis $\alpha$-entropy for all $\alpha>0$. It is convenient to change slightly the notation as follows. Let $X$ and $Y$ be discrete random variables with probabilities $\left\{p_{X}(x)\right\}$ and $\left\{p_{Y}(y)\right\}$, each supported on the $N$-point set $\Omega$. By $p_{X Y}(x, y)$ and $p_{X \mid Y}(x \mid y)$ we respectively denote the joint and conditional probabilities. The joint $\alpha$-entropy and the conditional $\alpha$-entropy are respectively defined as

$$
\begin{aligned}
H_{\alpha}(X, Y) & :=\frac{1}{1-\alpha}\left(\sum_{x, y} p_{X Y}(x, y)^{\alpha}-1\right), \\
H_{\alpha}(X \mid Y) & :=\sum_{y} p_{Y}(y)^{\alpha} H_{\alpha}(X \mid y),
\end{aligned}
$$

where $H_{\alpha}(X \mid y)=(1-\alpha)^{-1}\left(\sum_{x} p_{X \mid Y}(x \mid y)^{\alpha}-1\right)$. Rewriting

$$
H_{\alpha}(X \mid y)=-\sum_{x} p_{X \mid Y}(x \mid y)^{\alpha} \ln _{\alpha} p_{X \mid Y}(x \mid y),
$$

we further obtain

$$
H_{\alpha}(X \mid Y)=-\sum_{x, y} p_{X Y}(x, y)^{\alpha} \ln _{\alpha} p_{X \mid Y}(x \mid y)
$$


due to $p_{Y}(y) p_{X \mid Y}(x \mid y)=p_{X Y}(x, y)$. We will follow the original scheme of derivation (see the classical text [13], section 6.2). The probability of error is expressed as

$$
P_{e}=\sum_{y} p_{Y}(y) q(e \mid y), \quad q(e \mid y)=1-p_{X \mid Y}(y \mid y)=\sum_{x \neq y} p_{X \mid Y}(x \mid y) .
$$

Lemma 4 For all $\alpha \in(0, \infty)$, there holds

$$
H_{\alpha}(X \mid Y) \leq \sum_{y} p_{Y}(y)^{\alpha} h_{\alpha}(q(e \mid y))+\ln _{\alpha}(N-1) \sum_{y} p_{Y}(y)^{\alpha} q(e \mid y)^{\alpha} .
$$

Proof. Using the expression for $q(e \mid y)$ and the definition (2.5), we write

$$
\begin{aligned}
& H_{\alpha}(X \mid y)=-p_{X \mid Y}(y \mid y)^{\alpha} \ln _{\alpha} p_{X \mid Y}(y \mid y)-\sum_{x \neq y} p_{X \mid Y}(x \mid y)^{\alpha} \ln _{\alpha} p_{X \mid Y}(x \mid y) \\
& =h_{\alpha}(q(e \mid y))+q(e \mid y)^{\alpha} \ln _{\alpha} q(e \mid y)-\sum_{x \neq y} p_{X \mid Y}(x \mid y)^{\alpha} \ln _{\alpha} p_{X \mid Y}(x \mid y) .
\end{aligned}
$$

Due to $q(e \mid y)=\sum_{x \neq y} p_{X \mid Y}(x \mid y)$ and the properties of $\alpha$-logarithm, the second and third terms in the right-hand side of (6.6) are combined as

$$
\begin{aligned}
& -\sum_{x \neq y} p_{X \mid Y}(x \mid y)^{\alpha} \ln _{\alpha} p_{X \mid Y}(x \mid y)+q(e \mid y) q(e \mid y)^{\alpha-1} \ln _{\alpha} q(e \mid y) \\
& =-\sum_{x \neq y} p_{X \mid Y}(x \mid y)^{\alpha}\left(\ln _{\alpha} p_{X \mid Y}(x \mid y)-p_{X \mid Y}(x \mid y)^{1-\alpha} q(e \mid y)^{\alpha-1} \ln _{\alpha} q(e \mid y)\right) \\
& =-\sum_{x \neq y} p_{X \mid Y}(x \mid y)^{\alpha}\left(\ln _{\alpha} p_{X \mid Y}(x \mid y)+p_{X \mid Y}(x \mid y)^{1-\alpha} \ln _{\alpha} \frac{1}{q(e \mid y)}\right) \\
& =-q(e \mid y)^{\alpha} \sum_{x \neq y} \frac{p_{X \mid Y}(x \mid y)^{\alpha}}{q(e \mid y)^{\alpha}} \ln _{\alpha} \frac{p_{X \mid Y}(x \mid y)}{q(e \mid y)} \leq q(e \mid y)^{\alpha} \ln _{\alpha}(N-1) .
\end{aligned}
$$

Here we used the identities $\ln _{\alpha}(1 / z)=-z^{\alpha-1} \ln _{\alpha} z$ (right before (6.7)) and $\ln _{\alpha}(\xi z)=\ln _{\alpha} \xi+\xi^{1-\alpha} \ln _{\alpha} z$ (right before (6.8)). Substituting (6.8) in (6.6) and further in (6.2), we obtain (6.5).

Theorem 3 Let random variables $X$ and $Y$ take values on the same finite set of cardinality $N$. For given value of the error probability $P_{e}$, the conditional entropy $H_{\alpha}(X \mid Y)$ is bounded from above as

$$
\begin{array}{lr}
H_{\alpha}(X \mid Y) \leq \frac{P_{e}^{\alpha}-\alpha P_{e}}{1-\alpha}+P_{e}^{\alpha} \ln _{\alpha}[N(N-1)] & (0<\alpha<1), \\
H_{\alpha}(X \mid Y) \leq h_{\alpha}\left(P_{e}\right)+P_{e}^{\alpha} \ln _{\alpha}(N-1) & (1<\alpha<\infty) .
\end{array}
$$

Proof. For $\alpha \in(0,1)$, we use the formula $h_{\alpha}(u)=(1-\alpha)^{-1}\left[u^{\alpha}+(1-u)^{\alpha}-1\right] \leq(1-\alpha)^{-1}\left(u^{\alpha}-\alpha u\right)$, which follows from (2.5) and the inequality

$$
1-(1-u)^{\alpha}=\int_{0}^{u} \alpha(1-t)^{\alpha-1} d t \geq \int_{0}^{u} \alpha d t=\alpha u .
$$

By these relations and $\xi_{y}=p_{Y}(y) q(e \mid y)$, the first sum in the right-hand side of (6.5) is no greater than

$$
\frac{1}{1-\alpha} \sum_{y} p_{Y}(y)^{\alpha}\left[q(e \mid y)^{\alpha}-\alpha q(e \mid y)\right] \leq \frac{1}{1-\alpha}\left(\sum_{y} \xi_{y}^{\alpha}-\alpha P_{e}\right),
$$

since $\sum_{y} p_{Y}(y)^{\alpha} q(e \mid y) \geq \sum_{y} p_{Y}(y) q(e \mid y)=P_{e}$. Using the Hölder inequality, we also obtain

$$
\max \left\{\sum_{y=1}^{N} \xi_{y}^{\alpha}: 0 \leq \xi_{y} \leq 1, \quad \sum_{y=1}^{N} \xi_{y}=P_{e}\right\}=N^{1-\alpha} P_{e}^{\alpha}
$$

which is reached for $\xi_{y}=P_{e} / N$. So the term $(1-\alpha)^{-1}\left(N^{1-\alpha} P_{e}^{\alpha}-\alpha P_{e}\right)$ is an upper bound on the right-hand side of (6.12). Adding this with the product of $\ln _{\alpha}(N-1)$ and (6.13) finally gives (6.9).

Using $p_{Y}(y)^{\alpha} \leq p_{Y}(y)$ for $\alpha>1$ and Jensen's inequality for the concave function (2.5), we have

$$
\sum_{y} p_{Y}(y)^{\alpha} h_{\alpha}(q(e \mid y)) \leq \sum_{y} p_{Y}(y) h_{\alpha}(q(e \mid y)) \leq h_{\alpha}\left(\sum_{y} p_{Y}(y) q(e \mid y)\right)=h_{\alpha}\left(P_{e}\right)
$$


and also $\sum_{y} p_{Y}(y)^{\alpha} q(e \mid y)^{\alpha} \leq\left(\sum_{y} p_{Y}(y) q(e \mid y)\right)^{\alpha}=P_{e}^{\alpha}$. By these two points, the inequality (6.5) immediately leads to $(6.10)$.

For $\alpha>1$, the inequality (6.10) with $P_{e}$ instead of $P_{e}^{\alpha}$ was derived in [16]. So we obtain an improvement of the known result. The formula (6.9) for $0<\alpha<1$ is a new bound. By construction, the bound (6.9) is not sharp. Nevertheless, this inequality is sufficiently exact for small values of $P_{e}$. The bounds (6.9) and (6.10) both show that $P_{e} \rightarrow 0$ implies $H_{\alpha}(X \mid Y) \rightarrow 0$. On the other hand, if $H_{\alpha}(X \mid Y)$ is large then the probability of making an error must be large as well. In this regard, the essence of our inequalities concurs with a typical use of the standard Fano inequality.

Uniform continuity is an important property of the von Neumann entropy. The first result in this issue was given by Fannes [12]. The Tsallis entropy itself [18, 47] and its partial sums [33] also obey the continuity property. Due to the classical Fano inequality, one can sharpen Fannes' bound (see theorem 3.8 and its proof of Csiszár in [30]). We shall now show that the Fano type inequalities (6.9) and (6.10) lead to the Fannes inequality in terms of Tsallis entropies. Using properties of the $\alpha$-logarithm, the joint entropy (6.1) can be rewritten as [16]

$$
H_{\alpha}(X, Y)=H_{\alpha}(X)+H_{\alpha}(Y \mid X)=H_{\alpha}(Y)+H_{\alpha}(X \mid Y) .
$$

Due to $H_{\alpha}(Y \mid X) \geq 0$, the difference $H_{\alpha}(X)-H_{\alpha}(Y) \leq H_{\alpha}(X \mid Y)$ is bounded from above by the right-hand side of (6.9) for $\alpha \in(0,1)$ and by the right-hand side of (6.10) for $\alpha \in(1,+\infty)$. For given distributions $\left\{p_{X}(x)\right\}$ and $\left\{p_{Y}(y)\right\}$, the joint probability mass function $p_{X Y}(x, y)$ can be built in such a way that

$$
P_{e}=D(X, Y)=\frac{1}{2} \sum_{x}\left|p_{X}(x)-p_{Y}(x)\right| .
$$

This follows from the coupling inequality (see, e.g., the book 26$]$ ). Setting $\left\{p_{X}(x)\right\}=\operatorname{spec}(\boldsymbol{\rho})$ and $\left\{p_{Y}(y)\right\}=\operatorname{spec}(\boldsymbol{\sigma})$, we then have $D(X, Y) \leq \mathrm{D}(\boldsymbol{\rho}, \boldsymbol{\sigma})$ (see, e.g., lemma 11.1 in [30]). When $N \geq 2$, the right-hand side of (6.9) increases with $P_{e}$ for all $0 \leq P_{e} \leq 1$, the right-hand side of (6.10) increases with $P_{e}$ for all $0 \leq P_{e} \leq(N-1) / N$. Replacing $D(X, Y)$ with larger $\mathrm{D}(\boldsymbol{\rho}, \boldsymbol{\sigma})$, we obtain the following result.

Theorem 4 Let $d$ be dimensionality of the Hilbert space and $\tau=\mathrm{D}(\boldsymbol{\rho}, \boldsymbol{\sigma})$; then

$$
\begin{array}{lr}
\left|\mathrm{H}_{\alpha}(\boldsymbol{\rho})-\mathrm{H}_{\alpha}(\boldsymbol{\sigma})\right| \leq \frac{\tau^{\alpha}-\alpha \tau}{1-\alpha}+\tau^{\alpha} \ln _{\alpha}[d(d-1)] & (0<\alpha<1, \quad 0 \leq \tau \leq 1), \\
\left|\mathrm{H}_{\alpha}(\boldsymbol{\rho})-\mathrm{H}_{\alpha}(\boldsymbol{\sigma})\right| \leq h_{\alpha}(\tau)+\tau^{\alpha} \ln _{\alpha}(d-1) & \left(1<\alpha, \quad 0 \leq \tau \leq \frac{d-1}{d}\right) .
\end{array}
$$

The relation (6.18), when $\alpha>1$, is just the uniform estimate obtained by a direct method in [47]. Incidentally, this method allow to derive the bound for all $0 \leq \tau \leq 1$. In the limit $\alpha \rightarrow 1$, the inequality (6.18) reproduces the statement of theorem 3.8 in [30]. For $\alpha \in(0,1)$, there also exists an inequality

$$
\left|\mathrm{H}_{\alpha}(\boldsymbol{\rho})-\mathrm{H}_{\alpha}(\boldsymbol{\sigma})\right| \leq \frac{(2 \tau)^{\alpha}-2 \tau}{1-\alpha}+(2 \tau)^{\alpha} \ln _{\alpha} d,
$$

provided that $\|\boldsymbol{\rho}-\boldsymbol{\sigma}\|_{1}=2 \tau \leq \alpha^{1 /(1-\alpha)}$. The bound (6.19) was actually proved in [18] for all $\alpha \in[0,2]$, but the bound (6.18) is better for $\alpha \geq 1$. Comparing (6.17) with (6.19), we see the following. In general, the bound (6.17) is weaker but covers all acceptable values $\tau \in[0,1]$ of the trace distance. The scope of (6.19) is restricted to the range $0 \leq 2 \tau \leq \alpha^{1 /(1-\alpha)}$. In low dimensions, however, the bound (6.17) can be better than (6.19). Say, for $d=2$ and $\alpha=1 / 2$ the bound (6.19) holds for $0 \leq \tau \leq 1 / 8$. In this range, the right-hand side of (6.19) is larger than the right-hand side of (6.17). Moreover, for sufficiently small $\tau$ the difference between the two bounds is up to $40 \%$. Thus, the bound (6.17) has some practical interest, at least in the primary qubit case.

\section{Acknowledgments}

The author thanks anonymous referee for very detailed comments.

[1] Audenaert, K.: Subadditivity of $q$-entropies for $q>1$, J. Math. Phys. 48, 083507 (2007) 
[2] Audenaert, K.M.R., Eisert, J.: Continuity bounds on the quantum relative entropy. J. Math. Phys. 46, 102104 (2005)

[3] Audenaert, K.M.R., Eisert, J.: Continuity bounds on the quantum relative entropy - II. J. Math. Phys. 52, 112201 (2011)

[4] Bhatia, R.: Positive Definite Matrices. Princeton University Press, Princeton (2007)

[5] Borland, L., Plastino, A.R., Tsallis, C.: Information gain within nonextensive thermostatistics. J. Math. Phys. 39, 64906501 (1998); 40, 2196(E) (1999)

[6] Bratteli, O., Robinson, D.W.: Operator Algebras and Quantum Statistical Mechanics 2. Equilibrium States. Models in Quantum Statistical Mechanics. Springer, Berlin (2002)

[7] Busch, P: Stochastic isometries in quantum mechanics. Math. Phys. Anal. Geom. 2, 83-106 (1999)

[8] Cai, L., Hansen, F.: Metric-adjusted skew information: convexity and restricted forms of superadditivity. Lett. Math. Phys. 93, 1-13 (2010)

[9] Csiszár, I.: A note on Jensen's inequality. Studia Sci. Math. Hungar. 1, 227-230 (1966)

[10] Csiszár, I.: Information-type measures of difference of probability distributions and indirect observations. Studia Sci. Math. Hungar. 2, 299-318 (1967)

[11] Csiszár, I.: Axiomatic characterizations of information measures. Entropy 10, 261-273 (2008)

[12] Fannes, M.: A continuity property of entropy density for spin lattice systems. Commun. Math. Phys. 31, 291-294 (1973)

[13] Fano, R.M.: Transmission of Information: a Statistical Theory of Communications. MIT Press and John Wiley \& Sons, New York (1961)

[14] Fedotov, A., Harremoës, P., Topsøe, F.: Refinements of Pinsker inequality. IEEE Trans. Inf. Theory 49, 1491-1498 (2003)

[15] Fuchs, C.A., van de Graaf, J.: Cryptographic distinguishability measures for quantum mechanical states. IEEE Trans. Inf. Theory 45, 1216-1227 (1999)

[16] Furuichi, S.: Information theoretical properties of Tsallis entropies. J. Math. Phys. 47, 023302 (2006)

[17] Furuichi, S., Yanagi, K., Kuriyama, K.: Fundamental properties of Tsallis relative entropy. J. Math. Phys. 45, 4868-4877 (2004)

[18] Furuichi, S., Yanagi, K., Kuriyama, K.: A generalized Fannes' inequality. J. Inequal. Pure Appl. Math. 8(1), 5 (2007)

[19] Gell-Mann, M., Tsallis, C., ed.: Nonextensive Entropy - Interdisciplinary Applications. Oxford University Press, Oxford (2004)

[20] Gilardoni, G.: On Pinsker's and Vajda's type inequalities for Csiszár's $f$-divergence. IEEE Trans. Inf. Theory 56, 5377-5386 (2010)

[21] Hansen, F.: The Wigner-Yanase entropy is not subadditive. J. Stat. Phys. 126, 643-648 (2007)

[22] Hiai, F., Mosonyi, M., Petz, D., Bény, C.: Quantum f-divergences and error correction. Rev. Math. Phys. 23, 691-747 (2011)

[23] Hiai, F., Ohya, M., Tsukada, M.: Sufficiency, KMS condition and relative entropy in von Neumann algebras. Pacific J. Math. 96, 99-109 (1981)

[24] Jenčová, A., Ruskai, M.B.: A unified treatment of convexity of relative entropy and related trace functions, with conditions for equality. Rev. Math. Phys. 22, 1099-1121 (2010)

[25] Kullback, S., Leibler, R.A.: On information and sufficiency. Ann. Math. Stat. 22, 79-86 (1951)

[26] Lindvall, T.: Lectures on the Coupling Method. John Wiley \& Sons, New York (1992)

[27] Majerník, V., Majerníková, E.: The determination of bounds of the $\beta$-entropic sum of two noncommuting observables. Rep. Math. Phys. 47, 381-392 (2001)

[28] Nielsen, M.A., Chuang, I.L.: Quantum Computation and Quantum Information. Cambridge University Press, Cambridge (2000)

[29] Petz, D.: Quasi-entropies for finite quantum systems. Rep. Math. Phys. 21, 57-65 (1986)

[30] Petz, D.: Quantum Information Theory and Quantum Statistics. Springer, Berlin (2008)

[31] Petz, D.: From $f$-divergence to quantum quasi-entropies and their use. Entropy 12, 304-325 (2010)

[32] Raggio, G.A.: Properties of $q$-entropies, J. Math. Phys. 36, 4785-4791 (1995)

[33] Rastegin, A.E.: Continuity and stability of partial entropic sums. Lett. Math. Phys. 94, 229-242 (2010)

[34] Rastegin, A.E.: Bounds on Shannon distinguishability in terms of partitioned measures. Quantum Inf. Process. 10, 123-138 (2011)

[35] Rastegin, A.E.: Entropic uncertainty relations for extremal unravelings of super-operators. J. Phys. A: Math. Theor. 44, $095303(2011)$

[36] Rastegin, A.E.: Some general properties of unified entropies. J. Stat. Phys. 143, 1120-1135 (2011)

[37] Rastegin, A.E.: Upper continuity bounds on the relative $q$-entropy for $q>1$. J. Math. Phys. 52, 062203 (2011)

[38] Rastegin A.E.: Notes on entropic uncertainty relations beyond the scope of Riesz's theorem. Int. J. Theor. Phys. 51, $1300-1315(2012)$

[39] Rastegin, A.E. Fano type quantum inequalities in terms of $q$-entropies. Quantum Inf. Process. DOI: 10.1007/s11128-0110347-6

[40] Rockafellar, R.T.: Convex Analysis. Princeton University Press, Princeton (1970)

[41] Ruskai, M.B.: Inequalities for quantum entropy: A review with conditions for equality. J. Math. Phys. 43, 4358-4375 (2002)

[42] Ruskai, M.B., Stillinger, F.M.: Convexity inequalities for estimating free energy and relative entropy. J. Phys. A: Math. Gen. 23, 2421-2437 (1990)

[43] Seiringer, R.: On the failure of subadditivity of the Wigner-Yanase entropy. Lett. Math. Phys. 80, 285-288 (2007)

[44] Tsallis, C.: Possible generalization of Boltzmann-Gibbs statistics. J. Stat. Phys. 32, 479-487 (1988)

[45] Uhlmann, A.: Relative entropy and the Wigner-Yanase-Dyson-Lieb concavity in an interpolation theory. Commun. Math. 
Phys. 54, 21-32 (1977)

[46] Vedral, V.: The role of relative entropy in quantum information theory. Rev. Mod. Phys. 74, $197-234$ (2002)

[47] Zhang, Z.: Uniform estimates on the Tsallis entropies. Lett. Math. Phys. 80, 171-181 (2007)

[48] Zozor, S., Portesi, M., Vignat, C.: Some extensions of the uncertainty principle. Physica A 387, 4800-4808 (2008) 